\title{
RNA Sequencing Reveals Novel LncRNAs/mRNAs Network Associated with Puerarin-mediated Inhibition of Cardiac Hypertrophy in Mice
}

\section{Shan Ye ( 94302055@qq.com )}

Zhujiang Hospital

\section{Wei-Yang Chen}

the second affiliated hospital of Guangzhou Medical university

\section{Caiwen Ou}

Dongguan Fifth People's Hospital: Binhaiwan Central Hospital of Dongguan

\section{Min-Sheng Chen}

Zhujiang Hospital

\section{Research Article}

Keywords: Long non-coding RNA, Message RNA, RNA sequencing, Cardiac hypertrophy, Puerarin

Posted Date: August 23rd, 2021

DOI: https://doi.org/10.21203/rs.3.rs-788283/v1

License: (c) (i) This work is licensed under a Creative Commons Attribution 4.0 International License. Read Full License 


\section{Abstract}

Background: Evidence has demonstrated that puerarin is a potential drug for the treatment of cardiac hypertrophy. However, the precise underlying molecular mechanisms of the protective effect of puerarin are still unclear. Here, we aimed to explore the regulatory mechanisms of IncRNAs/mRNAs in a cardiac hypertrophy mouse model after puerarin treatment.

Methods: A mouse model of cardiac hypertrophy was established by transverse aortic constriction (TAC). The echocardiography, tissue staining and western blot were used to examine the protective effect of puerarin. Then RNA sequencing (RNA-seq) was carried out to systematically analyze global gene expression. The target IncRNAs were confirmed using qRT-PCR. Moreover, a coding/non-coding gene coexpression (CNC) network was established to find the interaction of IncRNAs and mRNAs. The molecular functions, biological processes, molecular components and pathways of different expression mRNAs targeted by IncRNA were explored using Gene Ontology (GO) analysis and Kyto Encyclopedia of Genes and Genomes (KEGG) pathways analysis.

Results: Puerarin exhibited obvious inhibitory effect in cardiac hypertrophy in TAC model. RNA-seq analysis was performed to investigate the IncRNAs and mRNAs expression patterns of cardiomyocytes in sham and TAC groups treated with or without puerarin. RNA-seq identified that TAC upregulated 19 IncRNAs and downregulated 18 IncRNAs, which could be revised by puerarin treatment (Fold change $\geq 3$ and $P<0.05)$. Expression alterations of selected IncRNAs ENSMUST00000125726, ENSMUST00000143044 and ENSMUST00000212795 were confirmed by qRT-PCR. Pearson's correlation coefficients of co-expression levels suggested that there was interactive relationship between those 3 validated altered IncRNAs and 5,500 mRNAs ( $r>0.95$ or $r<-0.95)$. Those co-expressed mRNAs were enriched in some important biological processes such as vesicle-mediated transport, $\sin 3$ complex, and translation initiation factor activity. KEGG analyses suggested that those IncRNA-interacted mRNAs were enriched in RNA transport, ribosome biogenesis in eukaryotes and proteasome signaling pathway.

Conclusion: Puerarin may exert beneficial effects on cardiac hypertrophy through regulating the ENSMUST00000125726 /ENSMUST00000143044 / ENSMUST00000212795 -mRNAs network.

\section{Introduction}

It is demonstrated that left ventricular hypertrophy is one of the independent risk factor for cardiovascular events, and cardiomyocyte hypertrophy is the main pathological change of left ventricular hypertrophy[1]. However, there is no effective treatment for myocardial hypertrophy so far[2]. Therefore, it is urgent to find an effective treatment for cardiomyocyte hypertrophy. Puerarin (PUE) is an active component isolated from the Traditional Chinese Medicine Gegen[3]. Recently, it was found that puerarin could significantly inhibit cardiomyocyte hypertrophy. It was reported that puerarin prevented cardiac hypertrophy through regulating the AMPK,mTOR and Nrf2 pathway, suggesting that puerarin may be potential drug candidate for myocardial hypertrophy[4,5]. However, the effect of puerarin on cardiomyocyte hypertrophy and its 
precise underlying molecular mechanisms need further study before its clinical usage for myocardial hypertrophy.

Long noncoding RNA (IncRNA) is a kind of non-coding RNA with a length of more than 200 nucleotides[6]. Recently, the role of IncRNA in cardiovascular disease has attracted more and more attention. Many previous studies have found that IncRNA is abundant in the cardiovascular system, which is involved in the pathophysiological processes of heart development, myocardial remodeling, myocardial hypertrophy, cardiomyocyte apoptosis, and so on[7]. Although current studies have demonstrated that IncRNAs play an important part in the pathophysiological process of cardiac disease, whether IncRNAs are associated with the protective role of puerarin in myocardial hypertrophy remains unknown. In the present study, RNA sequencing (RNA-seq) was performed to systematically understand the function of IncRNAs in the pharmacological action of puerarin in myocardial hypertrophy.

\section{Materials And Methods}

\subsection{Study design and transverse aortic constriction (TAC)- induced myocardial hypertrophy mouse model}

Male C57BL/ 6 mice (aged 5 weeks old) used in this study were purchased from the Guangdong Medical Laboratory Animal Center (Guangzhou, China). The mice were fed a standard laboratory diet and housed under standard conditions. Two weeks later, mice were randomly divided into the following three groups: the sham group, TAC group and TAC + PUE group. The sham group mice received intraperitoneal (i.p.) injection of the saline and the surgical procedures without the constriction. TAC group mice received i.p. injection of the saline and the TAC procedures to induce cardiac hypertrophy. The TAC + PUE group mice received a daily i.p. injection of puerarin (Sigma Aldrich, MO, USA) 3 days before TAC and continued for 21 days after TAC. The doses administered for puerarin (100 mg/kg per day) were chosen based on previous studies[8].

TAC is widely used as a disease model of chronic ventricular hypertrophy[9]. Thereby, TAC was used to establish myocardial hypertrophy in mice as described in a previous study[10]. Briefly, following anesthetization with isoflurane (Henry Schein, Melville, NY, USA), the aortic arch was exposed after a midline incision in the anterior neck. The transverse aortic arch between the left common carotid artery and the brachiocephalic artery was chosen as the site of constriction. The aortic arch was constricted by tying a 6.0 nylon suture ligature against a 26-gauge needle. After rapid removal of the needle, an incomplete constriction was formed. The successful constriction of TAC was verified using trans-thoracic echocardiography. All procedures were carried out according to the Guide for the Care and Use of Laboratory Animals (National Institutes of Health, USA)[11].

\subsection{Organ Weight}

Body weight (BW) and tibia length ( $T L$ ) of each mouse were measured 21 days after the TAC procedure. The mice were sacrificed using cervical dislocation under aesthesia, and hearts were arrested in diastole 
with injection of potassium chloride. And then, the hearts were gained and heart weight $(\mathrm{HW})$ were measured. Heart weight to body weight ratio (HW/BW) and heart weight to tibia length ratios (HW/TL) were counted.

\subsection{Echocardiography}

After anesthetization with isoflurane, successful ligation was confirmed by color Doppler and pulsedwave Doppler scanning. Left ventricular posterior wall dimension (LVPWd) and interventricular enddiastolic septum thickness (IVSd) were measured by two-dimensional transthoracic echocardiography. The transthoracic echocardiography was performed by an experienced technician who was blinded to the study groups using an IE33 echocardiographic system (Philips Medical Systems, Leiden, the Netherlands).

\subsection{Hematoxylin-Eosin (HE) Staining}

After fixation with $10 \%$ formalin, the heart tissues were dehydrated through a serial alcohol gradient and embedded in paraffin wax blocks. And then, heart sections were stained with HE solution (Beyotime, China). The sections were examined under a light microscope (Nikon Technology Co., Ltd., Japan).

\subsection{Western Blot Analysis}

Western blot analysis was performed as described previously [12-14]. Briefly, the whole protein of the heart was extracted using radio-immunoprecipitation assay (RIPA) lysis buffer (Kaiji Company, Shen Zhen, China) with protease and phosphatase inhibitors (Kaiji Company, Shen Zhen, China). Protein samples were separated by sodium dodecylsulphate polyacrylamide gel electrophoresis (SDS-PAGE) and transferred to polyvinylidene difluoride (PVDF) membranes. The membranes were then incubated at $4^{\circ} \mathrm{C}$ overnight with the following primary antibodies: $\beta$-MHC (Bioworld Technology Inc, Louis Park, MN; BS70815, 1:1000) and GAPDH (Cell Signaling Technology Inc., Danvers, USA; \# 5174, 1:1000). After being washed three times with PBST, the membranes were incubated with the secondary antibodies for 1 hour at room temperature. Then, the signals were detected using the Imaging System (GE, Amersham Imager 600, GE, Piscataway, NJ, USA). The relative expression level of proteins was analyzed using Image-Pro Plus 6.0 (Media Cybernetics Inc., Bethesda, MD, USA).

\subsection{RNA extraction}

The mice were sacrificed at 21 days after TAC and hearts were harvested. Total RNA was isolated from heart tissues using Trizol reagent (TaKaRa, Tokyo, Japan). The concentration of the RNA samples was evaluated using a NanoDrop ND-1000 instrument (thermo fisher scientific, USA). The integrity of the RNA was assessed by electrophoresis on an agarose gel.

\subsection{RNA sequencing analysis}

Library preparation and Illumina sequencing analysis were performed as previous study[15]. Briefly, the RNA of heart tissue was used for library construction by KAPA Stranded RNA-seq library Prep Kit (Illumina, NEB, USA). Then, the library was sequenced by Illumina NovaSeq 6000 Sequencing system 
(Illumina, NEB, USA) according to the manufacturer's protocol. The RAN-seq experiment was completed by Kangcheng Biotechnology Co., Ltd (Shanghai, China). Raw sequence files have been deposited at NCBI's Gene Expression Omnibus (Accession code: GSE176244). Differentially expressed IncRNAs with statistical significance among the three groups were observed through Volcano Plot filtering. Hierarchical clustering was conducted to demonstrate the distinguishable IncRNAs expression pattern among the groups.

\subsection{Quantitative real-time polymerase chain reaction (qRT- PCR) analysis}

The IncRNA-seq results were further validated by qRT-PCR analysis as previous study[16]. Briefly, total RNA isolated from heart tissues was reverse transcribed to synthesize cDNA. Then, the qRT-PCR was performed by the QuantStudio5 Real-time PCR System (Applied Biosystems) with the $2 \times$ PCR Master Mix (Arraystar: AS-MR-006-5). Relative expression levels of IncRNAs were normalized with GAPDH. The sequences of primers used for amplification are shown in Table 1. The expression of IncRNAs was calculated using the $2^{-\Delta \Delta C t}$ method.

Table 1

Sequence of the primers for IncRNAs.

\begin{tabular}{|llll|}
\hline & Forward and reverse primer & Tm & Product length (bp) \\
& & $\left.\mathbf{(}^{\circ} \mathrm{C}\right)$ & \\
\hline GAPDH & F:5' CACTGAGCAAGAGAGGCCCTAT3' & 60 & 144 \\
& R:5' GCAGCGAACTTTATTGATGGTATT3' & & \\
\hline ENSMUST00000125726 & F:5'TCTAGTGTCTGTTCCCTACTCGTG3' & 60 & 196 \\
& R:5'GAGGGACACAGCCATCAACA3' & & \\
\hline ENSMUST00000155474 & F:5'CCGCCAGGAAGAAAGATGTT3' & 60 & 264 \\
& R:5'CTCTCAGTGGCTCACCTTCTATG3' & & \\
\hline ENSMUST00000143044 & F:5'CATGGGCTTCAGGGTCACTA3' & 60 & 105 \\
& R:5'CGGGGCTCCACATCCAAT3' & & \\
\hline ENSMUST00000212795 & F:5'CGTGCGGGCTCTTTAACTGT3' & 60 & 185 \\
& R:5'GGCTCGGGACAACAACATTC3' & & \\
\hline Tm: annealing-temperature. bp: base pair. & & \\
\hline
\end{tabular}

2.9. Construction of the IncRNA-mRNA co-expression network 
The coding/non-coding gene co-expression (CNC) network profile was constructed according to validated altered IncRNAs and their related mRNAs. The CNC was established through the weighted gene coexpression network analysis (WGCNA) using the R package WGCNA (v1.69) [17]. The co-expression network between mRNA and IncRNA was constructed utilizing Cytoscape version 2.8.1 software (The Cytoscape Consortium, San Diego, CA, USA) based on the Pearson correlation analysis of IncRNA and mRNA $(r>0.95$ or $r<-0.95)$.

\subsection{Gene function analysis}

To explore the function of the selected genes in the co-expression network, Gene Ontology (GO) enrichment analysis and Kyoto Encyclopedia of Genes and Genomes (KEGG) pathway enrichment analysis for the targets genes were implemented using Database for Annotation, Visualization and Integrated Discovery (DAVID)[18, 19].

\subsection{Statistical analysis}

All results were presented as the mean \pm standard deviation. Statistical analysis was performed using GraphPad Prism 7.0 software (San Diego, CA, USA). One-way ANOVA followed by Tukey's post hoc analysis was used to test the differences among groups. A $P$ value less than 0.05 was considered statistically significant.

\section{Results}

\subsection{Puerarin inhibited TAC induced-cardiac hypertrophy in mics.}

As shown in Fig. 1A, the TAC group had significant high heart weight/body weight (HW/BW) ratio and heart weight/tibial length $(\mathrm{HW} / \mathrm{TL})$ ratio compared with sham group. Puerarin treatment resulted in significant reduction of HW/BW and HW/TL ratio compared with TAC group. Echocardiography demonstrated that TAC resulted the increasing of LVPWd and IVSd, which were significantly reversed by puerarin (Fig. 1B). Besides, HE staining analysis of cardiac sections showed that the increased myocyte area in TAC mice was minimised by treatment with puerarin (Fig. 1C). Protein levels of $\beta$-MHC was detected by western blotting. As expected, puerarin significantly reduced the $\beta$-MHC expression compared with TAC group (Fig. 1D).

\subsection{IncRNA expression profiles and validation.}

RNA-seq was used to assess the expression levels of IncRNAs in heart samples of the sham, TAC and TAC + PUE groups. Overall, 6355 IncRNAs were identified by RNA-seq among the groups. We identified 133 significantly dysregulated IncRNAs in the TAC group compared with sham group: 56 were upregulated, while 77 were down-regulated $(\geq 3.0$ fold, $P<0.05$ ). In addition, 27 IncRNAs were significantly up-regulated and 24 were down-regulated in TAC + PUE group compared with TAC group $(\geq 3.0$ fold, $P<$ 
0.05) (Fig. 2A and B). Then, venn diagrams and heat map showed the number of IncRNAs commonly expressed among the groups. Nineteen IncRNAs elevated in the TAC group were reduced by puerarin treatment. Eighteen IncRNAs downregulated in the TAC group were upregulated by puerarin treatment (Fig. 2C and Fig. 3A). Therefore, a total of 37 IncRNAs were altered significantly among the groups and was selected for further study. Among them, 30 IncRNAs (81\%) were exon sense-overlapping, 3 IncRNAs (8\%) were intergenic, 2 IncRNAs (5\%) were intron sense-overlapping, and 2 IncRNAs (5\%) were bidirectional (Fig. 3B).

To validate the RNA-seq results, four IncRNAs were selected for the qRT-PCR analysis. The qRT-PCR results were basically consistent with the RNA-seq data. There was significant difference among the groups in ENSMUST00000125726, ENSMUST00000143044 and ENSMUST00000212795 $(P<0.05)$ (Fig. 4).

\subsection{IncRNA-mRNA network analysis}

To investigate the underlying regulating mechanisms of IncRNAs in cardiac hypertrophy and the therapeutic target of puerarin, we analyzed the co-expression of IncRNAs and protein-coding genes by WGCNA. We calculated Pearson's correlation coefficients between the expression levels of 3 verified IncRNAs and 14,280 differentially expressed protein-coding gene. We used the 3 validated IncRNAs and the top 120 mRNAs co-expression with them to construct a IncRNA -mRNA visualization network using Cytoscape. These IncRNAs comprised multiple common target mRNA (Fig. 5A). The heat map showed the expression of the first 40 association mRNAs based on the degree of correlation (Fig. 5B).

Next, we carried out a functional enrichment analysis of the candidate IncRNA target genes using DAVID software[20]. GO analysis found that these target mRNAs were enriched in some biological processes such as vesicle-mediated transport, $\sin 3$ complex, and translation initiation factor activity (Fig. 6A-C). KEGG analyses showed that those IncRNA-interacted mRNAs were enriched in RNA transport, ribosome biogenesis in eukaryotes and proteasome signaling pathway (Fig. 6D).

\section{Discussion}

In the present study, we comprehensively investigated the role of IncRNAs in the cardioprotection of puerarin through RNA-sEq. RNA-seq revealed that TAC upregulated 19 IncRNAs and downregulated 18 IncRNAs, which were reversed by puerarin treatment. Among them, three novel IncRNA ENSMUST00000125726, ENSMUST00000143044 and ENSMUST00000212795 were verified by qRT-PCR. This data suggests that puerarin protects cardiomyocytes possibly through regulating IncRNAs.

It was found that IncRNAs were significantly altered in the mouse model of cardiac hypertrophy, suggesting that IncRNAs might play an important role in the pathogenesis of cardiac hypertrophy[21]. Previous studies demonstrated critical roles of particular IncRNAs such as Mhrt, Chaer and MEG3 in TAC mice. Those IncRNAs are proved to be candidates in the pathologic progression of hypertrophy cardiomyopathy[22, 23]. We found that ENSMUST00000125726, ENSMUST00000143044 and 
ENSMUST00000212795 were novel IncRNAs contributing to cardiac hypertrophy. Moreover, we found that those dysregulated IncRNAs could be reversed by puerarin. Thus, those dysregulated IncRNAs might be promising therapeutic targets to suppress cardiac hypertrophy[21]. Importantly, those three IncRNAs might be involved in puerarin-mediated inhibition of cardiac hypertrophy in mice. The study provides some new therapeutic targets to prevent or reverse myocardial hypertrophy.

The IncRNAs play critical roles in gene expression regulation in diverse biological process[24]. Several researches have showed that IncRNA-gene interactions are closely related to the occurrence and development of myocardial hypertrophy[22]. Therefore, it is important to explore the regulatory mechanism of IncRNA-mRNA network involved in myocardial hypertrophy and identify the target of puerarin[25]. We used the co-expression of IncRNAs and protein-coding genes from the RNA-seq to investigate the underlying regulating mechanisms of IncRNAs in cardiac hypertrophy and the therapeutic target of puerarin. A CNC network was built for the 3 verified IncRNAs and 120 of differentially expressed mRNAs based on the degree of correlation. The result demonstrated that those 120 coding genes potentially regulated by ENSMUST00000125726, ENSMUST00000143044 and ENSMUST00000212795. Through the Gene Ontology analysis, we found that these target mRNAs were enriched in biological processes such as vesicle-mediated transport, $\sin 3$ complex, and translation initiation factor activity in cardiomyocyte. We also analyzed the enriched KEGG pathway of those target genes. Three signaling pathways, including RNA transport, ribosome biogenesis in eukaryotes and proteasome signaling pathways, were significantly enriched from IncRNA-mRNAs network. Although previous studies have found that mitogen-activated protein kinase (MAPK), calcineurin-nuclear factor of activated T cells (NFAT), insulin-like growth factor-I (IGF-I)-phosphatidylinositol 3-kinase (PI3K)-AKT and glucose metabolic pathways are mainly responsible for the pathogenesis of myocardial hypertrophy[26-28]. This study found three undiscovered pathways regulated by IncRNAs were involved in the pathogenesis of myocardial hypertrophy. Moreover, those pathways might be the target of puerarin in the treatment of cardiac hypertrophy.

In conclusion, our study used deep RNA-seq analysis to reveal puerarin could reverse myocardial hypertrophy by regulating IncRNAs. In addition, we revealed some IncRNA-mRNA network involved in therapeutic effect of puerarin. This study provides novel insights into our understanding of the pathogenesis of cardiac hypertrophy and proves the therapeutic role of puerarin in preventing or reversing cardiac hypertrophy. The limitation of this study is the lack of functional assessment of the identified IncRNA and mRNAs. Further mechanisms study will provide us comprehensive understanding of puerarin pharmacological activities, which will be helpful for the development of clinical patient treatment and clinical practice guidelines.

\section{Declarations}

\section{Conflict of interest}

All authors declare that there are no conflicts of interest. 


\section{Funding}

This work was partly supported by Medical Scientific Research Foundation of Guangdong Province, China (grant No. A2020106).

\section{References}

1. Hutchings DC, Anderson SG, Caldwell JL, Trafford AW (2018) Phosphodiesterase-5 inhibitors and the heart: compound cardioprotection? Heart 104:1244-1250. doi:10.1136/heartjnl-2017-312865

2. Rodrigues PG, Leite-Moreira AF, Falcao-Pires I (2016) Myocardial reverse remodeling: how far can we rewind? Am J Physiol Heart Circ Physiol 310:H1402-H1422. doi:10.1152/ajpheart.00696.2015

3. Zhang $L$ (2019) Pharmacokinetics and drug delivery systems for puerarin, a bioactive flavone from traditional Chinese medicine. Drug Deliv 26:860-869. doi:10.1080/10717544.2019.1660732

4. Cai SA, Hou N, Zhao GJ, Liu XW, He YY, Liu HL, Hua YQ, Li LR, Huang Y, Ou CW, Luo CF, Chen MS (2018) Nrf2 Is a Key Regulator on Puerarin Preventing Cardiac Fibrosis and Upregulating Metabolic Enzymes UGT1A1 in Rats. Front Pharmacol 9:540. doi:10.3389/fphar.2018.00540

5. Liu B, Wu Z, Li Y, Ou C, Huang Z, Zhang J, Liu P, Luo C, Chen M (2015) Puerarin prevents cardiac hypertrophy induced by pressure overload through activation of autophagy. Biochem Biophys Res Commun 464:908-915. doi:10.1016/j.bbrc.2015.07.065

6. Yang Q, Han Y, Liu P, Huang Y, Li X, Jia L, Zheng Y, Li W (2020) Long Noncoding RNA GAS5 Promotes Osteogenic Differentiation of Human Periodontal Ligament Stem Cells by Regulating GDF5 and p38/JNK Signaling Pathway. Front Pharmacol 11:701. doi:10.3389/fphar.2020.00701

7. Gomes CPC, Schroen B, Kuster GM, Robinson EL, Ford K, Squire IB, Heymans S, Martelli F, Emanueli C, Devaux Y, Action EU-CC (2020) Regulatory RNAs in Heart Failure. Circulation 141:313-328. doi:10.1161/CIRCULATIONAHA.119.042474

8. Chen G, Pan SQ, Shen C, Pan SF, Zhang XM, He QY (2014) Puerarin inhibits angiotensin Il-induced cardiac hypertrophy via the redox-sensitive ERK1/2, p38 and NF-kappaB pathways. Acta Pharmacol Sin 35:463-475. doi:10.1038/aps.2013.185

9. Bosch L, de Haan JJ, Bastemeijer M, van der Burg J, van der Worp E, Wesseling M, Viola M, Odille C, El Azzouzi H, Pasterkamp G, Sluijter JPG, Wever KE, de Jager SCA (2020) The transverse aortic constriction heart failure animal model: a systematic review and meta-analysis. Heart Fail Rev. doi:10.1007/s10741-020-09960-w

10. Meng G, Liu J, Liu S, Song Q, Liu L, Xie L, Han Y, Ji Y (2018) Hydrogen sulfide pretreatment improves mitochondrial function in myocardial hypertrophy via a SIRT3-dependent manner. Br J Pharmacol 175:1126-1145. doi:10.1111/bph.13861

11. National Research Council (U.S.). Committee for the Update of the Guide for the Care and Use of Laboratory Animals., Institute for Laboratory Animal Research (U.S.) and National Academies Press 
(U.S.) (2011) Guide for the care and use of laboratory animals. 8th edn. National Academies Press,, Washington, D.C. pp. $x x v, 220 p$

12. Lin SP, Li W, Winters A, Liu R, Yang SH (2018) Artemisinin Prevents Glutamate-Induced Neuronal Cell Death Via Akt Pathway Activation. Front Cell Neurosci 12:108. doi:10.3389/fncel.2018.00108

13. Peng X, Wang Y, Li H, Fan J, Shen J, Yu X, Zhou Y, Mao H (2019) ATG5-mediated autophagy suppresses NF-kappaB signaling to limit epithelial inflammatory response to kidney injury. Cell Death Dis 10:253. doi:10.1038/s41419-019-1483-7

14. Lin SP, Wei JX, Hu JS, Bu JY, Zhu LD, Li Q, Liao HJ, Lin PY, Ye S, Chen SQ, Chen XH (2021) Artemisinin improves neurocognitive deficits associated with sepsis by activating the AMPK axis in microglia. Acta Pharmacol Sin. doi:10.1038/s41401-021-00634-3

15. Li H, Wei X, Yang J, Dong D, Huang Y, Lan X, Plath M, Lei C, Qi X, Bai Y, Chen H (2017) Developmental transcriptome profiling of bovine muscle tissue reveals an abundant GosB that regulates myoblast proliferation and apoptosis. Oncotarget 8:32083-32100. doi:10.18632/oncotarget.16644

16. Lin SP, Hu J, Wei JX, Ye S, Bu J, Xu W, Chen S, Wu Y, Wu G, Zhu L, Lin PY, Chen XH (2020) Silencing of circFoxO3 Protects HT22 Cells from Glutamate-Induced Oxidative Injury via Regulating the Mitochondrial Apoptosis Pathway. Cell Mol Neurobiol 40:1231-1242. doi:10.1007/s10571-02000817-2

17. Langfelder P, Horvath S (2008) WGCNA: an R package for weighted correlation network analysis. BMC Bioinformatics 9:559. doi:10.1186/1471-2105-9-559

18. Huang da W, Sherman BT, Lempicki RA (2009) Systematic and integrative analysis of large gene lists using DAVID bioinformatics resources. Nat Protoc 4:44-57. doi:10.1038/nprot.2008.211 nprot.2008.211 [pii]

19. Lin SP, Ye S, Long Y, Fan Y, Mao HF, Chen MT, Ma QJ (2016) Circular RNA expression alterations are involved in OGD/R-induced neuron injury. Biochem Biophys Res Commun 471:52-56. doi:10.1016/j.bbrc.2016.01.183

20. Chen S, Wu Y, Qin X, Wen P, Liu J, Yang M (2021) Global gene expression analysis using RNA-seq reveals the new roles of Panax notoginseng Saponins in ischemic cardiomyocytes. J Ethnopharmacol 268:113639. doi:10.1016/j.jep.2020.113639

21. Wang L, Wang J, Li G, Xiao J (2020) Non-coding RNAs in Physiological Cardiac Hypertrophy. Adv Exp Med Biol 1229:149-161. doi:10.1007/978-981-15-1671-9_8

22. Liu L, Zhang D, Li Y (2020) LncRNAs in cardiac hypertrophy: From basic science to clinical application. J Cell Mol Med 24:11638-11645. doi:10.1111/jcmm.15819

23. Xu Y, Luo Y, Liang C, Zhang T (2020) LncRNA-Mhrt regulates cardiac hypertrophy by modulating the miR-145a-5p/KLF4/myocardin axis. J Mol Cell Cardiol 139:47-61. doi:10.1016/j.yjmcc.2019.12.013

24. He W, Wei D, Cai, Chen S, Li S, Chen W (2018) Altered Long Non-Coding RNA Transcriptomic Profiles in Ischemic Stroke. Hum Gene Ther 29:719-732. doi:10.1089/hum.2017.064

25. Zhang L, Zeng H, Wang JH, Zhao H, Zhang B, Zou J, Yoshida S, Zhou Y (2020) Altered Long Noncoding RNAs Involved in Immunological Regulation and Associated with Choroidal 
Neovascularization in Mice. Int J Med Sci 17:292-301. doi:10.7150/ijms.37804

26. Avila-Medina J, Mayoral-Gonzalez I, Galeano-Otero I, Redondo PC, Rosado JA, Smani T (2020) Pathophysiological Significance of Store-Operated Calcium Entry in Cardiovascular and Skeletal Muscle Disorders and Angiogenesis. Adv Exp Med Biol 1131:489-504. doi:10.1007/978-3-03012457-1_19

27. Tran DH, Wang ZV (2019) Glucose Metabolism in Cardiac Hypertrophy and Heart Failure. J Am Heart Assoc 8:e012673. doi:10.1161/JAHA.119.012673

28. Heineke J, Molkentin JD (2006) Regulation of cardiac hypertrophy by intracellular signalling pathways. Nat Rev Mol Cell Biol 7:589-600. doi:10.1038/nrm1983

\section{Figures}

(A)

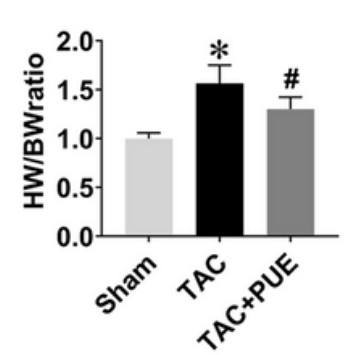

(B)

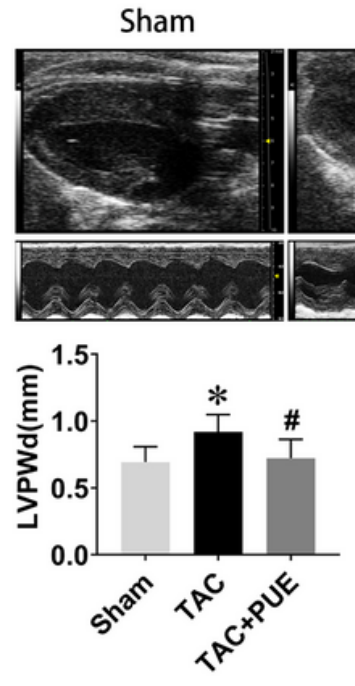

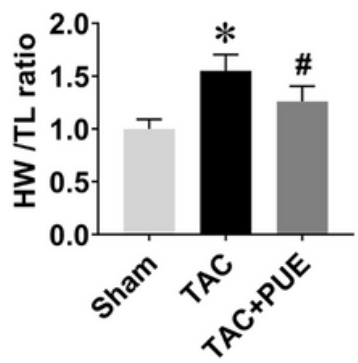

TAC
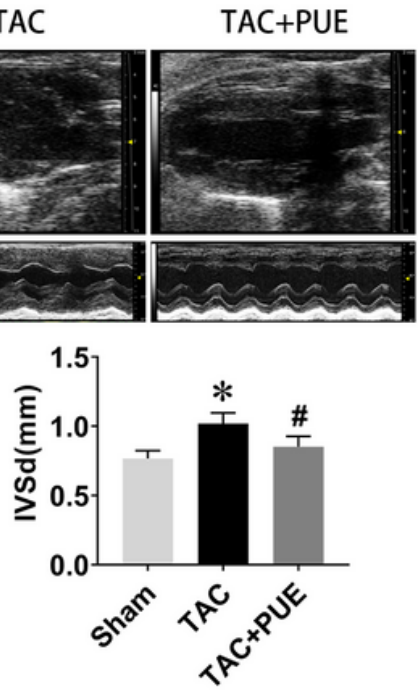

(C)

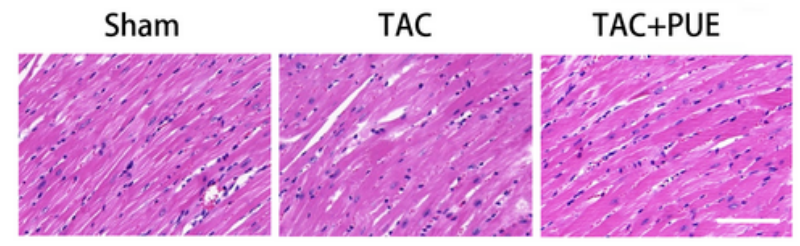

(D)
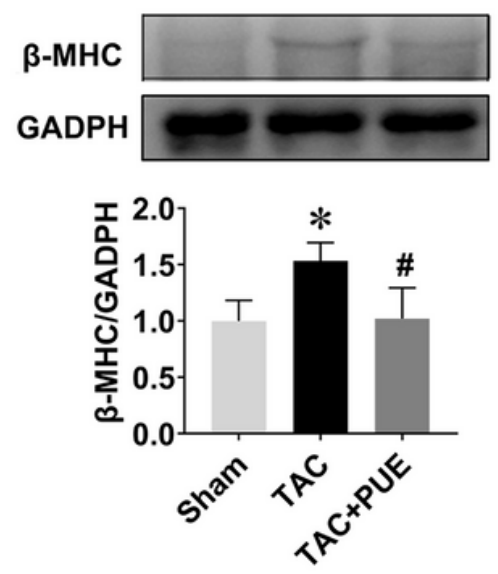

\section{Figure 1}

Puerarin decreased the cardiac hypertrophy induced by TAC in mice. (A) Heart weight/body weight (HW/BW) ratio and heart weight/tibial length $(\mathrm{HW} / \mathrm{TL})$ ratio from sham and TAC groups treated with or without puerarin $(n=5)$. (B) Left ventricular posterior wall dimension (LVPWd) and interventricular enddiastolic septum thickness (IVSd) measured by echocardiography $(n=5)$. (C) Representative images of HE staining (Scale bar=100 $\mu \mathrm{m}$ ). (D) Protein levels of $\beta-M H C$ was detected by western blotting $(n=3)$. ${ }^{*} P<0.05$ versus the sham group. \# $P<0.05$ versus the TAC group. 
(A)

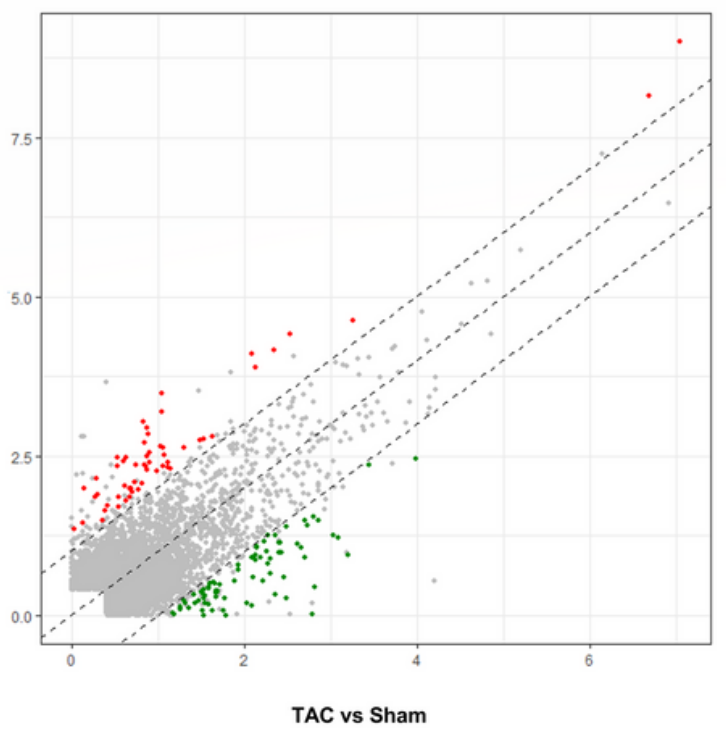

(B)

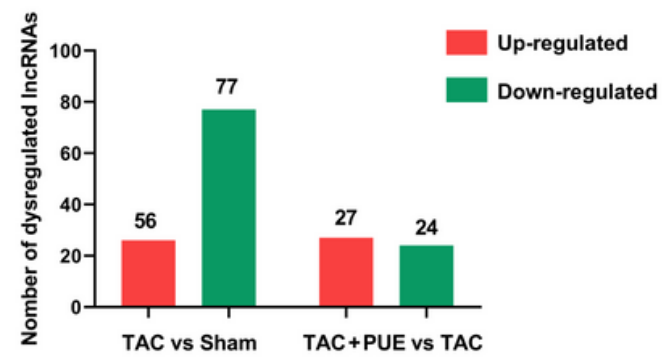

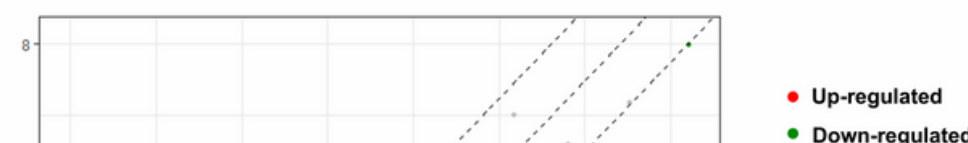

- Down-regulated

(C)
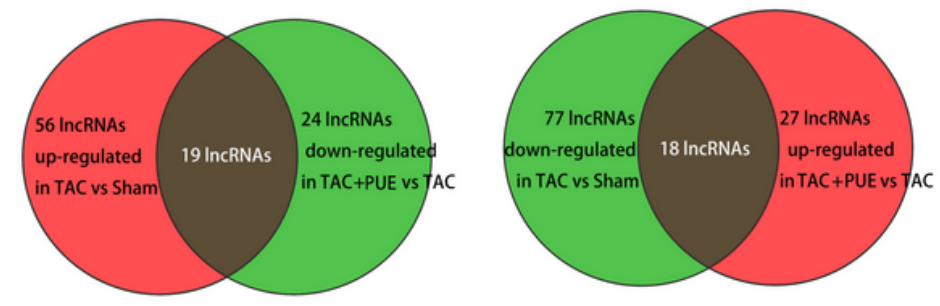

\section{Figure 2}

Analysis of IncRNAs expression using RNA-seq data. (A) Scatter plot displaying differentially expressed IncRNAs among the groups. (B) Number of up- and down-regulated IncRNAs in each group. The $x$ - and $y$ axes show the fold change (log2 transformed) in IncRNAs expression. Red points denote up-regulated IncRNAs and green points represent down-regulated IncRNAs (Fold change greater than 3-fold and $\mathrm{P}<$ 0.05). Grey points represent IncRNAs with no significant differences. (C) Venn diagrams showed the number of IncRNAs commonly expressed among the groups. Nineteen IncRNAs that were elevated in the TAC group were reduced by puerarin treatment. Eighteen IncRNAs that were downregulated in the TAC group were upregulated by puerarin treatment. 
(A)

\begin{tabular}{|c|c|c|c|c|c|c|c|c|c|}
\hline \multicolumn{10}{|l|}{ ENSMUST00000125726 } \\
\hline \multirow{2}{*}{\multicolumn{10}{|c|}{$\begin{array}{l}\text { ENSMUST00000155474 } \\
\text { ENSMUST00000210692 }\end{array}$}} \\
\hline & & & & & & & & & \\
\hline \multicolumn{10}{|l|}{ ENSMUST00000189045 } \\
\hline \multicolumn{10}{|l|}{ ENSMUST00000182537 } \\
\hline \multicolumn{10}{|l|}{ ENSMUST00000182520 } \\
\hline \\
\hline \multirow{2}{*}{\multicolumn{10}{|c|}{$\begin{array}{l}\text { ENSMUST00000146117 } \\
\text { ENSMUSTO0000143073 }\end{array}$}} \\
\hline & & & & & & & & & \\
\hline \multicolumn{10}{|l|}{ ENSMUST00000139623 } \\
\hline \multicolumn{10}{|l|}{$\begin{array}{l}\text { ENSMUST00000138071 } \\
\text { ENSMUST00000135456 }\end{array}$} \\
\hline ENSMUST00000135456 & & & & & & & & & \\
\hline \multicolumn{10}{|l|}{ ENSMUST00000134861 } \\
\hline \multicolumn{10}{|l|}{ ENSMUST00000133683 } \\
\hline \multirow{2}{*}{\multicolumn{10}{|c|}{$\begin{array}{l}\text { ENSMUST00000132276 } \\
\text { ENSMUST00000127679 }\end{array}$}} \\
\hline & & & & & & & & & \\
\hline \multicolumn{10}{|l|}{ ENSMUSTO0000127423 } \\
\hline \multicolumn{10}{|l|}{ ENSMUST00000106779 } \\
\hline \multicolumn{10}{|l|}{ ENSMUST00000059704 } \\
\hline \multirow{2}{*}{\multicolumn{10}{|c|}{$\begin{array}{l}\text { ENSMUST } \\
\text { ENSSMUS }\end{array}$}} \\
\hline \multirow{2}{*}{\multicolumn{10}{|c|}{$\begin{array}{l}\text { ENSMUST00000212795 } \\
\text { ENSMUSTO0000218372 }\end{array}$}} \\
\hline & & & & & & & & & \\
\hline \multicolumn{10}{|l|}{ ENSMUST00000193235 } \\
\hline \multicolumn{10}{|l|}{ ENSMUST00000150194 } \\
\hline \multirow{2}{*}{\multicolumn{10}{|c|}{$\begin{array}{l}\text { ENSMUST00000148239 } \\
\text { ENSMUST00000147635 }\end{array}$}} \\
\hline \multirow{2}{*}{\multicolumn{10}{|c|}{ ENSMUST00000143464 }} \\
\hline & & & & & & & & & \\
\hline \multicolumn{10}{|l|}{ ENSMUST00000143168 } \\
\hline \multicolumn{10}{|l|}{ ENSMUST00000142342 } \\
\hline \multicolumn{10}{|l|}{ ENSMUST00000135831 } \\
\hline \multicolumn{10}{|l|}{ ENSMUST00000134793 } \\
\hline \multirow{2}{*}{\multicolumn{10}{|c|}{ ENSMUST00000134040 }} \\
\hline & & & & & & & & & \\
\hline ENSMUST00000123890 & & & & & & & & & \\
\hline ENSMUST00000101522 & & & & & & & & & \\
\hline ENSMUST00000071254 & & & & & & & & & \\
\hline ENSMUST00000197507 & & & & & & & & & \\
\hline
\end{tabular}

(B)

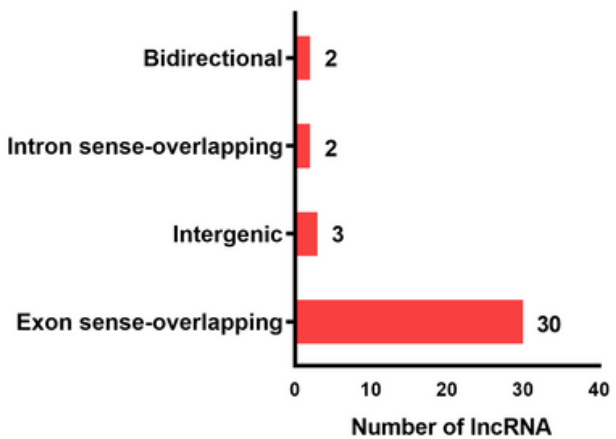

\section{Figure 3}

Altered IncRNAs expressions among the groups. (A) There were 19 IncRNAs up-regulated in TAC group, which was reversed by puerarin treatment. Eighteen IncRNAs were down-regulated in TAC group and were up-regulated after puerarin treatment (Fold change $\geq 3$ and $P<0.05$ ). Up-regulated IncRNAs are shown in red, and down-regulated IncRNAs are shown in green. (B) Type and proportion of the differentially expressed IncRNAs. 
(A)

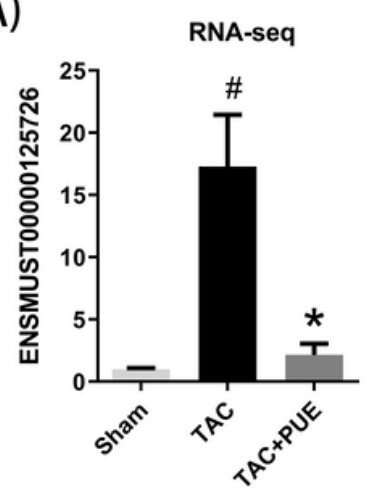

(C)

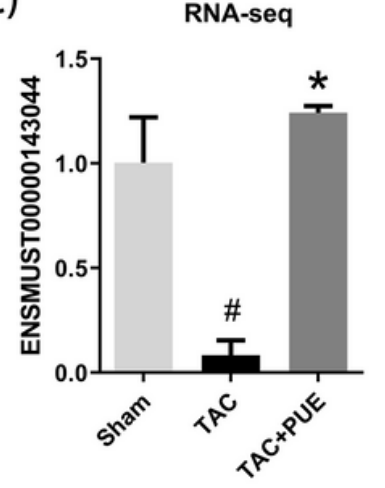

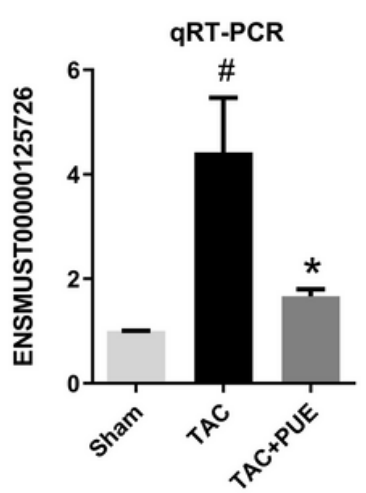

qRT-PCR

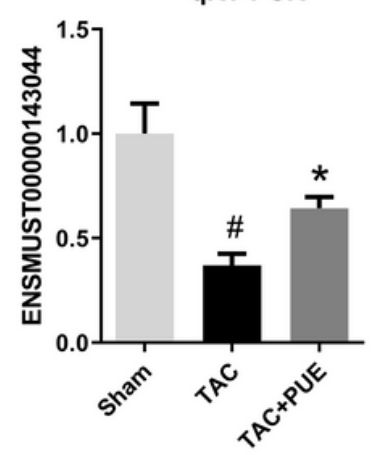

(B)
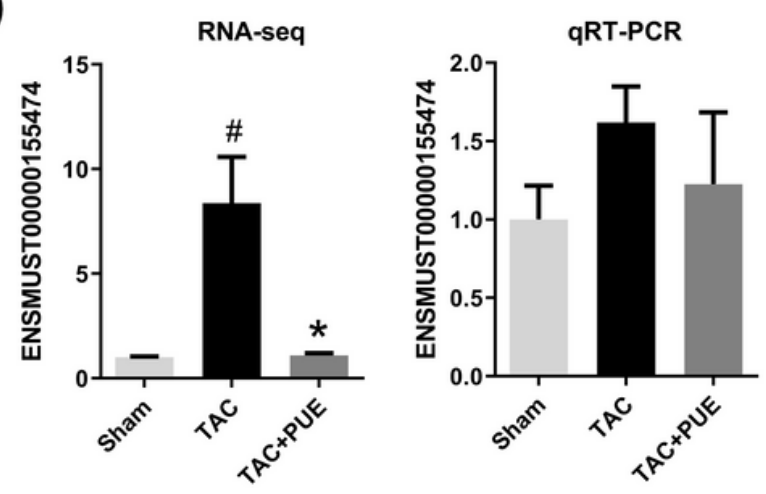

(D)

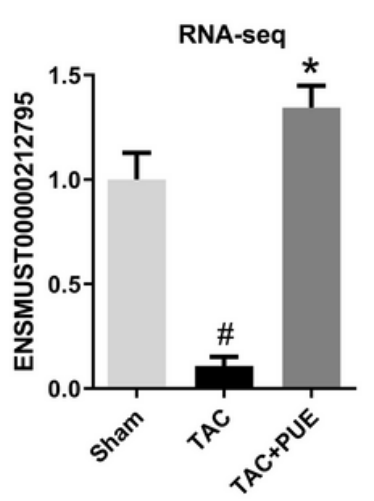

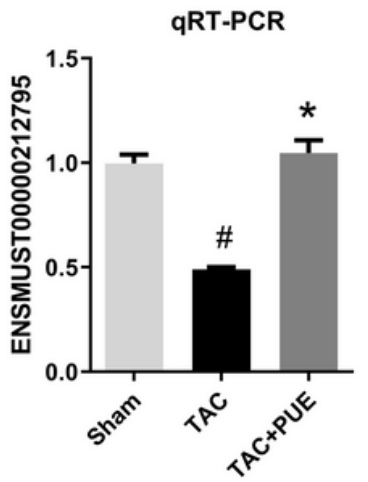

Figure 4

The quantitative real-time PCR (qRT-PCR) validation of four differentiation expressed IncRNAs with the largest fold changes in RNA-seq. The RNA-seq and qRT-PCR results among the groups in ENSMUST00000125726 (A), ENSMUST00000155474 (B), ENSMUST00000143044 (C) and ENSMUST00000212795 (D). * $P<0.05$ versus the sham group. \# $P<0.05$ versus the TAC group. 
(A)

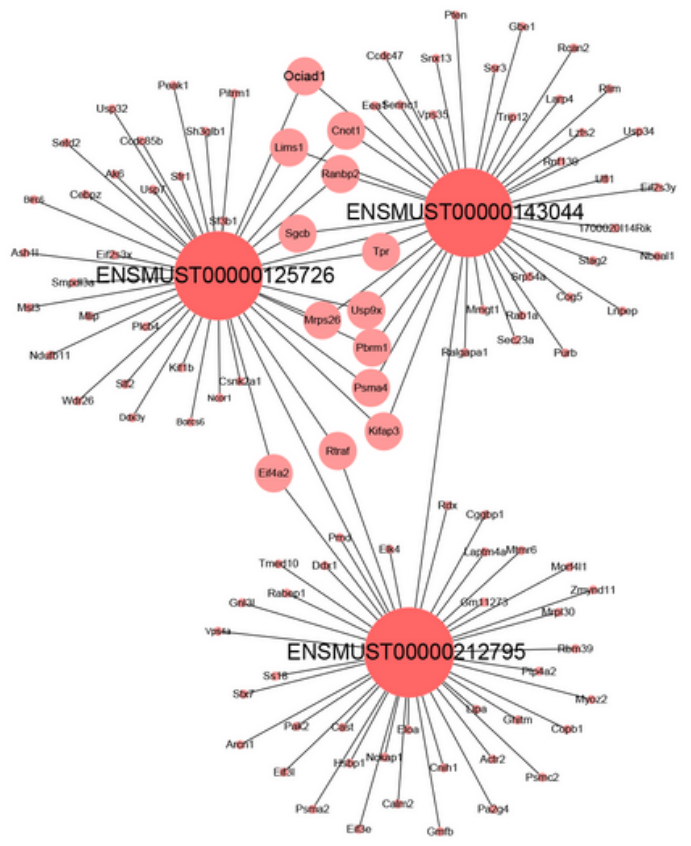

(B)

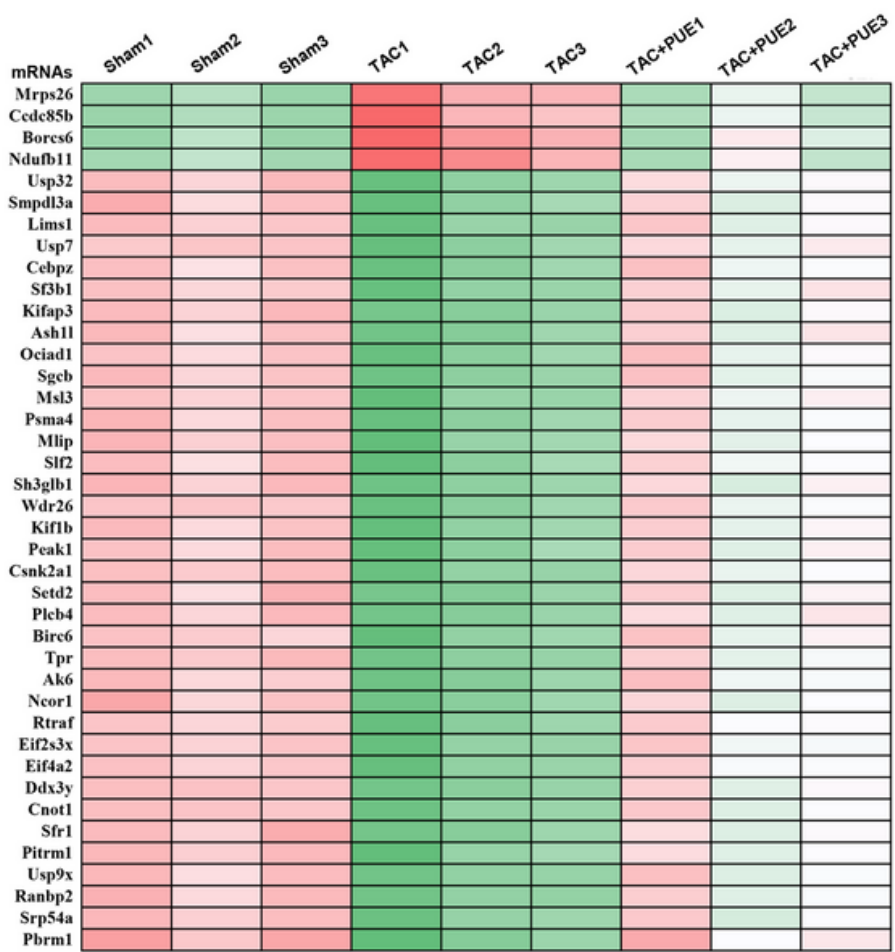

\section{Figure 5}

CNC networks of validated IncRNAs. $\triangle A \otimes T h$ co-expression network profiles of IncRNAs and mRNAs based on 3 validated IncRNAs. (B) Heat map analysis of the mRNAs co-expression with validated IncRNAs.

(A)

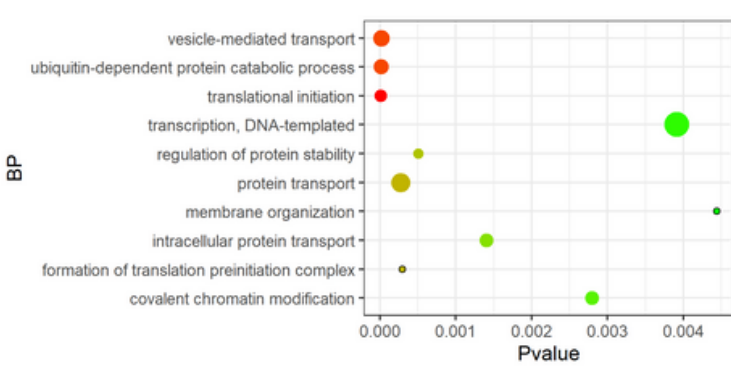

(C)

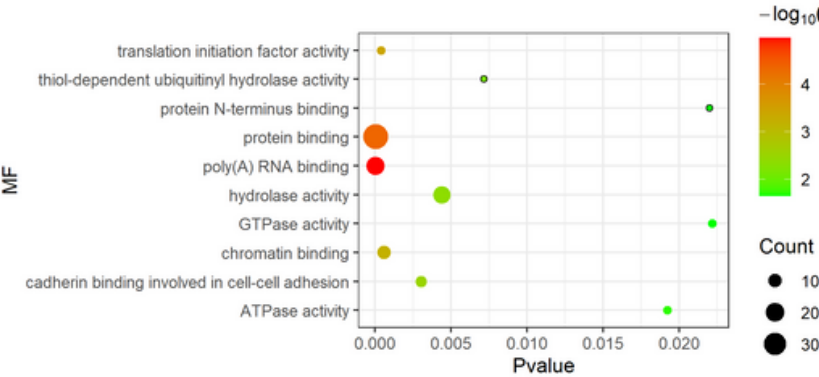

(B)

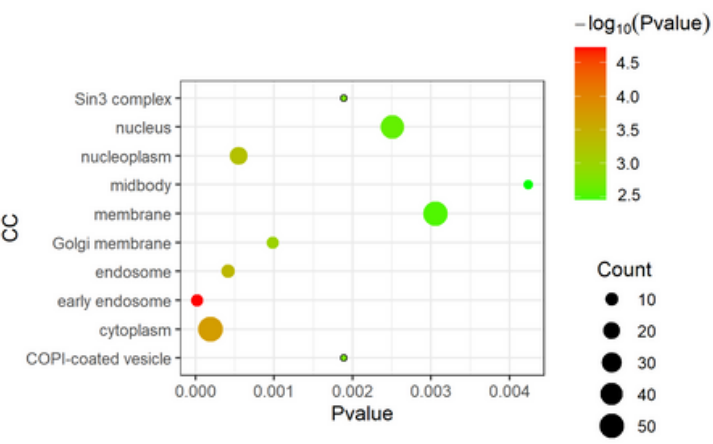

(D)

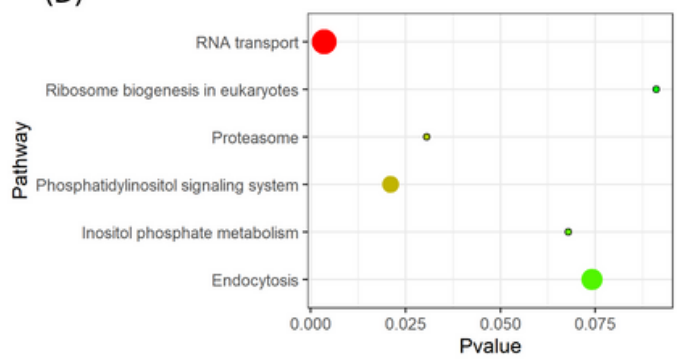

$-\log _{10}$ (Pvalue)

2.0

1.5

Count

- 3

$\begin{array}{r}14 \\ \hline\end{array}$

\section{Figure 6}


The GO and KEGG analyses of target mRNAs. (A) The top 10 significant biological process (BP) of GO analyses. (B) The top 10 significant cellular component (CC) of GO analyses. (C) The top 10 significant molecular function (MF) of GO analyses. (D) The significant enriched pathways by KEGG pathway analysis.

\section{Supplementary Files}

This is a list of supplementary files associated with this preprint. Click to download.

- GADPH.tif

- renamedc44c7.tif 